\title{
一种 $\beta, \beta, \beta$-二氟磑-1-苯基乙醇类化合物的简便合成方法
}

\author{
黄国志任洁郑笑笑吴范宏* 吴晶晶* \\ (上海应用技术大学化学与环境工程学院 上海 201418)
}

\begin{abstract}
摘要 以二氟硔代丙酮类化合物为原料, 利用硼氢化钠选择性还原得到高产率的二氟碘代醇类化合物。该合成方法具 有反应条件温和，操作简单，产率高等特点，为合成更多的二氟取代醇类化合物试剂提供了简便的途径.

关键词 二氟硒代苯乙酮; 硼氢化钠; 二氟碘代苯乙醇; 选择性还原
\end{abstract}

\section{A Convenient Synthesis of $\beta, \beta$-Difluoro- $\beta$-iodo-1-phenylethan-1-ols}

\author{
Huang, Guozhi Ren, Jie Zheng, Xiaoxiao Wu, Fanhong* Wu, Jingjing* \\ (School of Chemical and Environmental Engineering, Shanghai Institute of Technology, Shanghai 201418)
}

\begin{abstract}
The synthesis of $\alpha, \alpha, \alpha$-iodo-difluoromethyl alcohols via the high selective reduction of iododifluoromethyl ketones by $\mathrm{NaBH}_{4}$ in methanol at $0{ }^{\circ} \mathrm{C}$ was reported. The reaction has some advantages, such as mild condition, simple synthetic route, and high yield. A convenient way to synthesize more difluoromethylation agents was provided.

Keywords iodo-difluoromethyl ketones; $\mathrm{NaBH}_{4}$; iodo-difluoromethyl alcohol; selective reduction
\end{abstract}

苯基乙醇类片段广泛存在于天然产物及具有生物 活性的化合物结构中，是有机化学中常见的一类结构片 段. 如图 1 所示的化合物 $\mathbf{A}$ 结构片段，被用于治疗哮喘， 包括哮喘发作、运动引起的支气管收缩和慢性阻塞性肺 病 $^{[1]}$. 化合物 $\mathbf{B}$ 用于治疗脚趾灰指甲 ${ }^{[2]}$. 化合物 $\mathbf{C}$ 临床 上主要用于癌症疼痛、骨折或术后疼痛等各种急、慢性 疼痛 ${ }^{[3]}$ (图 1). 因此, 苯基乙醇类化合物合成方法研究一 直引起化学家的广泛关注.

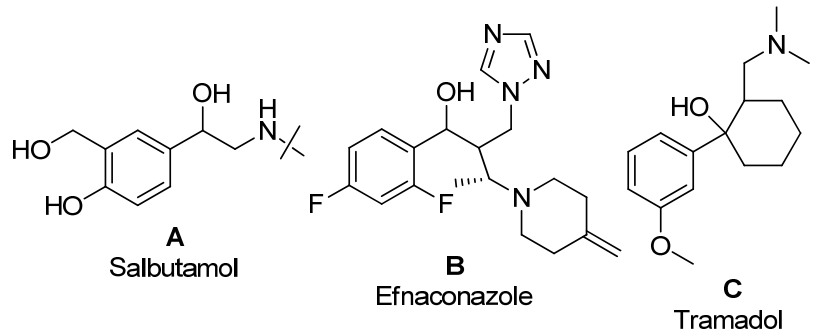

图 1 含有苯基乙醇类结构的化合物

Figure 1 High bioactive compounds containing phenylethanol motif

氟原子具有较高的电离电势和电负性, 具有极化率 较低, 原子半径较小等特点, 向有机分子中引入氟原子
通常对其性质具有很大的影响 ${ }^{[4]}$. 将含氟原子或含氟基 团引入到具有活性的母核结构中成为新药设计与开发 的一个重要研究手段 ${ }^{[5]}$. 然而到目前为止, 很少关于含 氟苯基乙醇类片段的合成方法的报道，早在 2007 年， Zanda 等 ${ }^{[6]}$ 通过由氟乙酸盐三步法合成了丙烯酸 $\beta$-氟烷 基酯(Scheme 1a). 2012 年胡金波等 ${ }^{[7]}$ 报道了通过 JuliaKocienski 反应进行羰基化合物的碘/溴二氟甲基化 (Scheme 1b)，以高收率得到碘/溴二氟甲基化的甲醇类 化合物的片段, 为合成各种有用的含氟化合物开辟了新 的途径. 2014 年. Dilman 等 ${ }^{[8]}$ 报道了用二氟澳/碘甲基三 甲基硅烷进行醛的亲核溴代和碘代反应(Scheme 1c)，该 反应在四丁基铵/锂盐 $\left(\mathrm{Bu}_{4} \mathrm{NX} / \mathrm{LiX}, \mathrm{X}=\mathrm{Br}\right.$ 或 $\mathrm{I}$ )在丙腈中 的组合存在下进行，通过相应的硅试剂进行醛的亲核性 溴代和碘代二氟甲基化，是一种合成含氟苯基乙醇类化 合物的便利方法. 2015 年 Mikami 等 ${ }^{[9]}$ 通过烯烃与二氟丙 酮酸催化不对称合成了含二氟甲基的叔醇和氧杂环戊 烯(Scheme 1d)，实现了在二价钯络合物存在下烯烃与 亲电子试剂二氟丙酮酸的高对映选择性催化不对称反 应，得到二氟甲基取代的叔醇类化合物. 前期我们课题 组设计并合成了一系列的含 $\alpha, \alpha, \alpha-$ 二氟磑代酮类化合物,

\footnotetext{
* Corresponding authors. E-mail:wjj_693@163.com; wfh@sit.edu.cn Received May 30, 2019; revised July 28, 2019; published online August 7, 2019.

Project supported by the General Project of the National Natural Science Foundation of China (No. 21672151). 国家自然科学基金面上(No. 21672151)资助项目.
} 
并将其用于各类二氟甲基化反应 ${ }^{[10]}$. 我们好奇 $\alpha, \alpha, \alpha-$ 二 氟碘代酮类砌块是否也可以通过转化羰基来得到 $\beta, \beta, \beta$ 二氟碘代醇类化合物, 从而得到更多结构多样的苯乙醇 类的片段. 因此我们尝试用不同的还原剂对 $\alpha, \alpha, \alpha$-二氟 碘代酮类化合物进行选择性还原, 期望得到 $\beta, \beta, \beta$-二氟 碘代醇类化合物, 进一步拓展含二氟碘代底物和更多的 含二氟卤代的醇类化合物. 以本课题组新开发的 $\alpha, \alpha, \alpha-$ 二氟碘代酮类含氟砌块为底物, 选择硼氢化钠进行选择 性还原得到相应的 $\beta, \beta, \beta$-二氟碘代醇类化合物. 该反应 具有易操作、产率高、绿色实用等优点 (Scheme 1e), 为 氟代苯乙醇类化合物的合成提供了新的简便的方法.

Previou work

(a)

$$
\text { (a) }
$$

(b)

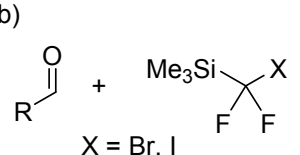

(c)

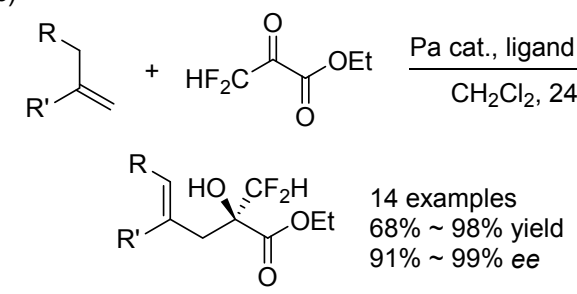

(d)

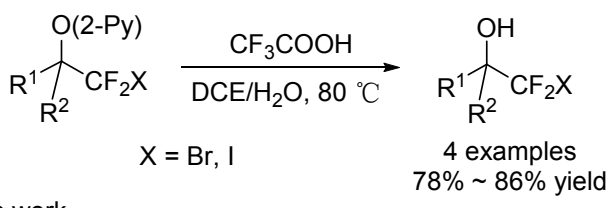

This work

(e)

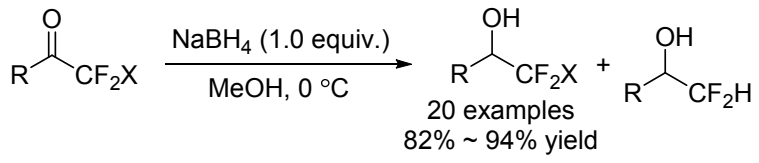

图式 1 苯基乙醇类化合物的合成方法

Scheme 1 Methods for synthesizing fluorine-containing phenylethanol compounds

在 $\alpha, \alpha, \alpha$-二氟碘代酮类化合物还原过程中, 因化合 物结构中含有可被还原的基团如羰基与碘元素, 理论上 存在 3 种不同的还原产物, 如 Scheme 2 所示. 因此, 我 们对在偕二氟基团存在情况下卤素与羰基基团竞争性 还原的结果如何产生了浓厚的兴趣, 并期望通过选择性 还原羰基基团得到相应的 $\beta, \beta, \beta$-二氟碘代醇类化合物，
该目标产物可作为含氟砌块与不同类型的底物反应合 成结构复杂多样的含 $\beta$-偕二氟基团的醇类片段化合物, 用于新药分子活性篮选工作.

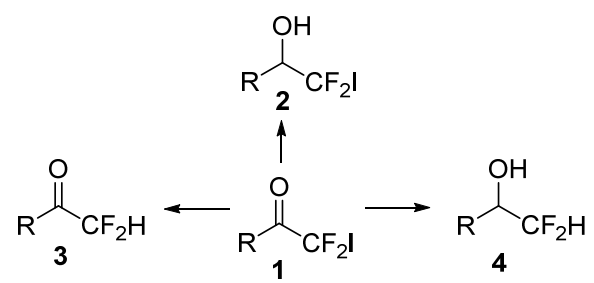

图式 $2 \alpha, \alpha, \alpha-$ 二氟碘代酮还原结果

Scheme 2 Reduction product of $\alpha, \alpha, \alpha$-iodo-difluoromethyl ketones

\section{1 结果与讨论}

选取 $\alpha, \alpha, \alpha$-二氟碘苯乙酮 (1a) 作为模板底物来探索 并优化合成 $\beta, \beta, \beta$-二氟碘-1-苯基乙醇(2a)的反应条件. 选择不同的还原试剂进行条件篎选, 得到的结果如表 1 所示. 采用不同的还原剂时，反应过程中均伴随着一定 量的 $\alpha, \alpha$-二氟苯乙酮 (3a) 和 $\beta, \beta$-二氟-1-苯基乙醇 $(4 \mathbf{a})$ 的 出现. 当选择实验室中最为常见的 $\mathrm{NaBH}_{4}$ 作为还原剂 时，它以一个很高的收率得到羰基被还原的产物 $\mathbf{2 a}$ (表 1, Entry 1), 表现出了很好的还原选择性. 接下来选用同 样有温和还原能力的 DIBAL-H ${ }^{[11]}$ 时, 也能以较好的收 率得到产物 $2 \mathbf{a}$, 也伴随着少量的羰基与碘同时被还原 的产物 $4 \mathrm{a}$ 生成(Entry 2). 当尝试还原能力较强的 $\mathrm{LiAlH}_{4}{ }^{[12]}$ 时(Entry 3)发现, 得到的产物较为复杂, 通过 $\mathrm{GC}$ 检测到有 $\mathbf{2 a}, \mathbf{3 a}, \mathbf{4 a}$ 三种不同的还原产物. 可见 $\mathrm{LiAlH}_{4}$ 作为还原剂对 $\alpha, \alpha, \alpha$-二氟碘苯乙酮的还原选择性 较差, 反应效果不好. 当采用 $\mathrm{H}_{2} / \mathrm{Pd} / \mathrm{C}$ 还原时, 都是原 料 1a, 没有转化为其他产物(Entry 4). 采用其他常见的

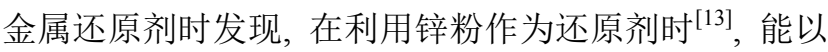
较高的转化率和较高的收率得到碘元素被还原的产物 3a (Entry 5). 选择红铝试剂 ${ }^{[14]}$ 作为还原剂时, 效果和 $\mathrm{LiAlH}_{4}$ 相似，同时得到三种不同的还原产物，而目标产 物 2a 收率却很少(Entry 6). 从这二者的还原效果来看, 推断在利用金属还原剂进行还原时，优先还原底物 $\mathbf{1 a}$ 中的碘，再进一步还原羰基官能团. 最后，选择超氢还 原剂 $\mathrm{LiEt}_{3} \mathrm{BH}$ 进行还原时 ${ }^{[15]}$, 发现主要得到自身缩合的 产物 4a' (Entry 7), 并非生成预料中的 2a, 3a 和 $\mathbf{4 a}$.

接下来, 对还原剂用量和温度对于反应的影响进行 了研究(表 2). 采用甲醇为反应溶剂, 在 $0{ }^{\circ} \mathrm{C}$ 时反应时. 目标产物 $2 \mathbf{a}$ 的产率会随着 $\mathrm{NaBH}_{4}$ 用量的逐渐增加而逐 渐减少, $\alpha$-碘和羰基均被还原的产物 $4 \mathrm{a}$ 的含量在逐渐增 加(表 2 , Entryies 1 4). 尝试将反应温度降至 $-10{ }^{\circ} \mathrm{C}$, 发现在 5 min 后 $1 \mathbf{a}$ 未能完全转化(Entry 5); 逐渐升高反 应温度, $0{ }^{\circ} \mathrm{C}$ 时条件原料能反应完全. 但是我们期待的 
表 1 不同还原剂得到的还原结果 ${ }^{a}$

Table 1 Reduction results of different reduction agent<smiles>O=C(c1ccccc1)C(F)(F)F</smiles>

1a<smiles>O=C(c1ccccc1)C(F)(F)C(F)(F)C(F)(F)C(F)(F)C(F)(F)F</smiles>

$3 a$

\begin{tabular}{clccccc}
\hline \multirow{2}{*}{ Entry } & \multirow{2}{*}{ Reduction (equiv.) } & \multirow{2}{*}{ Conv./\% } & \multicolumn{5}{c}{ Yield $^{b} / \%$} \\
\cline { 3 - 7 } & & & $\mathbf{2 a}$ & $\mathbf{3 a}$ & $\mathbf{4 a}$ & $\mathbf{4 a}^{\prime}$ \\
\hline 1 & $\mathrm{NaBH}_{4}(1.0)$ & 100 & 98.9 & 0 & 1.1 & \\
2 & $\mathrm{DIBAL} \mathrm{H}(1.0)$ & 100 & 81.3 & 0 & 18.7 & \\
3 & $\mathrm{LiAlH}_{4}(0.4)$ & 100 & 32.4 & 45.4 & 22.2 & \\
4 & $10 \% \mathrm{Pd} / \mathrm{C} / \mathrm{H}_{2}(0.1)$ & 0 & 0 & 0 & 0 & 0 \\
5 & $\mathrm{Zn}(1.0)$ & 100 & 0.6 & 98.5 & 0.9 & \\
6 & Red-Al (1.0) & 100 & 8.8 & 37.4 & 53.8 & \\
\hline 7 & LiEt $_{3} \mathrm{BH}(1.2)$ & 100 & 0.8 & 0 & 0.3 & 98.9 \\
\hline
\end{tabular}

${ }^{a}$ Reaction condition: $\mathbf{1 a}(1 \mathrm{mmol}), \mathrm{MeOH}(10 \mathrm{~mL})$ at room temperature for 0.5 $\mathrm{h},{ }^{b}$ Base on GC.

4a':<smiles>O=C(c1ccccc1)C(O)(C(F)(F)F)C(F)(F)c1ccccc1</smiles>

表 2 还原剂用量和温度对反应的影响 ${ }^{a}$

Table 2 Effects of reductant amount and temperature on the reaction

\begin{tabular}{ccccccc}
\hline \multirow{2}{*}{ Entry } & \multirow{2}{*}{$\mathrm{NaBH}_{4} /$ equiv. } & \multirow{2}{*}{ Temp. $/{ }^{\circ} \mathrm{C}$} & \multirow{2}{*}{ Conv. $/ \%$} & \multicolumn{3}{c}{ Yield $^{b} / \%$} \\
\cline { 5 - 7 } & & & $\mathbf{2 a}$ & $\mathbf{3 a}$ & 4a \\
\hline 1 & 0.5 & 0 & 95.4 & 94.0 & 0 & 1.4 \\
2 & 1.0 & 0 & 100 & 98.9 & 0 & 1.1 \\
3 & 2.0 & 0 & 100 & 84.2 & 0 & 15.8 \\
4 & 3.0 & 0 & 100 & 71.5 & 0 & 28.5 \\
5 & 1.0 & -10 & 99.8 & 98.3 & 0 & 1.5 \\
6 & 1.0 & r.t. & 100 & 86.2 & 0 & 13.8 \\
7 & 1.0 & Reflux & 100 & 86.3 & 0.1 & 13.6 \\
\hline${ }^{a}$
\end{tabular}

${ }^{a}$ Reaction condition: 1a $(1 \mathrm{mmol}), \mathrm{MeOH}(10 \mathrm{~mL})$ for 5 min. ${ }^{b}$ Base on GC.

选择性产物 2a 的量逐渐减少, 4a 的量在逐渐增加并趋 向于稳定(Entries 6,7). 结果表明, 选择还原剂 $\mathrm{NaBH}_{4}$ 1.0 equiv., 反应温度 $0{ }^{\circ} \mathrm{C}$, 为反应最佳还原剂和温度 (Entry 2).

随后对研究反应时间和反应溶剂对于反应的影响 进行了研究(表 3). 将反应时间从 $5 \mathrm{~min}$ 延长到 $30 \mathrm{~min}$, 2a 的收率从 $98.9 \%$ 降低到 $89.0 \%$, 而且副产物 4a 的收率 从 $1.1 \%$ 升高到 $11.0 \%$ (表 3, Entry 1). 再延长反应时间至 $60 \mathrm{~min}$ 后, 2a 的收率较 $30 \mathrm{~min}$ 时收率略微降低, 2a 的收 率整体呈下降趋势(Entry 2), 可见延长反应时间并不能 提高 2a 产率. 当反应溶剂由 $\mathrm{MeOH}$ 变为另一种溶剂 THF 后, 发现结果较为复杂, 得到 3 种不同的还原产物 的混合物, 且目标产物 $\mathbf{2 a}$ 的收率最低, 从而说明该溶剂
并不适合该反应(Entriy 3).

表 3 反应时间和溶剂对反应的影响 ${ }^{a}$

Table 3 Effects of time and solvent on the reaction ${ }^{a}$

\begin{tabular}{ccccccc}
\hline \multirow{2}{*}{ Entry } & \multirow{2}{*}{ Solvent } & \multirow{2}{*}{ Time/min } & \multirow{2}{*}{ Conv./\% } & \multicolumn{3}{c}{ Yield $^{b} \%$} \\
\cline { 5 - 7 } & & & & $\mathbf{2 a}$ & $\mathbf{3 a}$ & 4a \\
\hline 1 & $\mathrm{MeOH}$ & 30 & 100 & 89.0 & 0 & 11.0 \\
2 & $\mathrm{MeOH}$ & 60 & 100 & 88.4 & 0 & 11.6 \\
3 & $\mathrm{THF}$ & 5 & 100 & 22.7 & 40.9 & 36.4 \\
\hline
\end{tabular}

${ }^{a}$ Reaction condition: 1a $(1 \mathrm{mmol}), \mathrm{NaBH}_{4}(1 \mathrm{mmol}), \mathrm{MeOH}(10 \mathrm{~mL})$ at $0{ }^{\circ} \mathrm{C}$ ${ }^{b}$ Base on GC.

综上所述, 最佳的选择性还原的反应条件是, $\mathrm{NaBH}_{4}$ 为还原试剂, 还原剂用量为 1.0 equiv. 溶剂为 $\mathrm{MeOH}$, 在 $0{ }^{\circ} \mathrm{C}$ 下反应.

在获得最佳反应条件后, 对反应底物进行了普适性 考察, 结果如表 4 所示. 从表 4 可知, 当芳环上无取代或 者在对位有甲基、甲氧基、氯、溴、三氟甲基等供电子 或吸电子取代基, 或者是杂环噻吩取代的官能团时, 都 能以优异的还原选择性和产率得到相应的目标产物 (2a $\sim 2 f ， 2 i)$; 但是当芳环上带有氟原子、荎基官能团、 4-苯基、4-苯基醚和含有不饱和脂肪族类取代底物时, 同时生成了相应的 $\alpha$-碘被还原的产物 $(4 \mathrm{~g}, 4 \mathrm{~h}, 4 \mathrm{j}, 4 \mathrm{l}, 4 \mathrm{~m}$, 4n). 这可能是由于这些基团的存在对于碘的活性产生 了一定的影响, 使碘在反应的过程中离去, 从而生成了 碘被还原的产物, 但也能以中等收率得到目标产物. 值 得一提的是，该方法不仅对二氟碘取代芳基酮类底物有 较好的适用性，而且对二氟碘取代脂肪族酮类底物也有 很好的还原选择性，以 $88 \%$ 的收率得到了目标产物 $\mathbf{2 k}$. 该方法同样对含有不饱和脂肪族类取代底物也有较高 的还原选择性, 得到了目标产物 2n, 进一步验证了该方 法的适用性较广.

在硼氢化钠存在下 $\alpha, \alpha, \alpha$-溴二氟苯乙酮和 $\alpha, \alpha, \alpha$-氯 二氟苯乙酮两个底物的选择性还原结果见 Scheme 3. 结 果表明, 两个底物均能以较好的还原选择性和较高的收 率得到羰基还原产物 20 和 $2 p$. 因此说明卤素原子对反 应的选择性影响不大，在该反应体系中均不会被硼氢化 钠还原，再次验证了该方法的普适性.

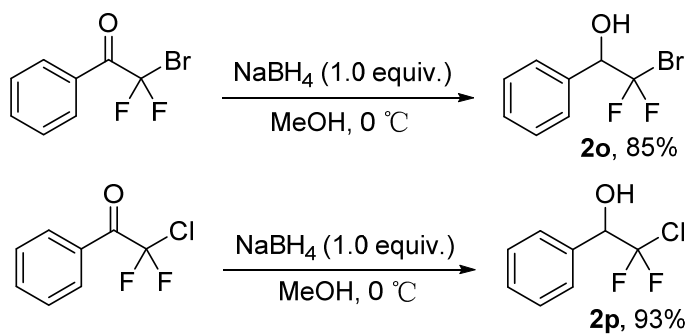

图式 $3 \alpha, \alpha, \alpha$-溴二氟苯乙酮和 $\alpha, \alpha, \alpha$-氯二氟苯乙酮的还原反 应考察

Scheme 3 Study on reduction reaction of $\alpha, \alpha, \alpha$-bromodifluoroacetophenone with $\alpha, \alpha$-bromofluoroacetophenone 
表 $4 \alpha, \alpha, \alpha$-碘二氟苯乙酮类化合物的底物拓展 ${ }^{a}$

Table 4 Substrate scope of $\alpha, \alpha, \alpha$-iododifluoroacetophenone compounds

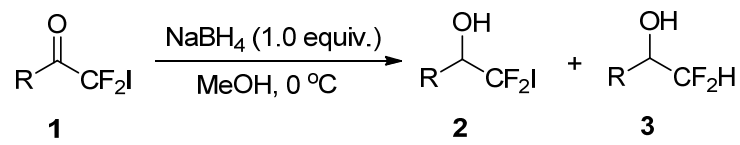<smiles>OC(c1ccccc1)C(F)(F)F</smiles>

2a, $94 \%$<smiles>OC(c1ccc(C(F)(F)F)cc1)C(F)(F)F</smiles>

2f, $86 \%$<smiles>OC(c1cccs1)C(F)(F)F</smiles>

2i, $90 \%$<smiles>Cc1ccc(C(O)C(F)(F)F)cc1</smiles>

2b, $93 \%$<smiles>COc1ccc(C(O)C(F)(F)F)cc1</smiles>

2c, $93 \%$<smiles>OC(c1ccc(Cl)cc1)C(F)(F)F</smiles>

2d, $92 \%$<smiles>OC(c1ccc(Br)cc1)C(F)(F)F</smiles>

2e, $91 \%$<smiles>OC(c1ccc(F)cc1)C(Cl)(Cl)Cl</smiles>

$2 \mathrm{~g}, 82 \%$<smiles>CC(F)(F)C(O)c1ccc(F)cc1</smiles>

4g, $9 \%$<smiles>OC(c1ccccc1F)C(F)(F)F</smiles>

2h, $83 \%$<smiles>CCCC(C)C(O)c1ccccc1F</smiles>

4h, $8 \%$<smiles>CC(C)C(F)(F)C(O)c1ccc2ccccc2c1</smiles>

$4 \mathrm{j}, 9 \%$<smiles>OC(CCc1ccccc1)C(F)(F)Cl</smiles>

2k, $88 \%$<smiles>OC(c1ccc(-c2ccccc2)cc1)C(F)(F)F</smiles>

2I, $85 \%$<smiles>OC(c1ccc(-c2ccccc2)cc1)C(F)(F)C(F)(F)F</smiles>

4I, $15 \%$<smiles>CC(C)C(O)c1ccc(Oc2ccc(C(F)(F)F)cc2)cc1</smiles>

$2 \mathrm{~m}, 84 \%$

$4 \mathrm{~m}, 16 \%$<smiles>OC(C=Cc1ccccc1)C(F)(F)F</smiles>

2n, $60 \%$<smiles>OC(/C=C/c1ccccc1)C(F)(F)F</smiles>

$4 n, 24 \%$

${ }^{a}$ Reaction condition: $1(1 \mathrm{mmol}), \mathrm{NaBH}_{4}(1 \mathrm{mmol}), \mathrm{MeOH}(10 \mathrm{~mL})$ at $0{ }^{\circ} \mathrm{C}$ for $5 \mathrm{~min}$. Isolated yields.

此外，探索了在该条件下，澳取代的单氟苯乙酮类 化合物选择还原性效果，如表 5 所示. 结果表明，当芳 环上无取代基或者在对位有甲基、甲氧基和氟等供电子 或吸电子取代基时, 都能以优异的选择性和产率得到相 应的目标产物 $(\mathbf{5 a} \sim \mathbf{5 d})$. 再次证明该方法对 $\alpha, \alpha, \alpha$-碘二 氟苯乙酮、 $\alpha, \alpha, \alpha$-溴二氟苯乙酮、 $\alpha, \alpha, \alpha$-氯二氟苯乙酮和 $\alpha, \alpha$-溴氟苯乙酮底物均有很高的选择还原性, 而对其 $\alpha$ 位卤原子无任何影响, 因此用 $\beta$-偕二氟取代醇类片段进 一步与不同的底物反应, 得到结构复杂多样的新颖含氟 化合物.

表 $5 \alpha, \alpha$-溴氟苯乙酮的底物拓展 ${ }^{a}$

Table 5 Substrate scope of $\alpha, \alpha$-bromofluoroacetophenone
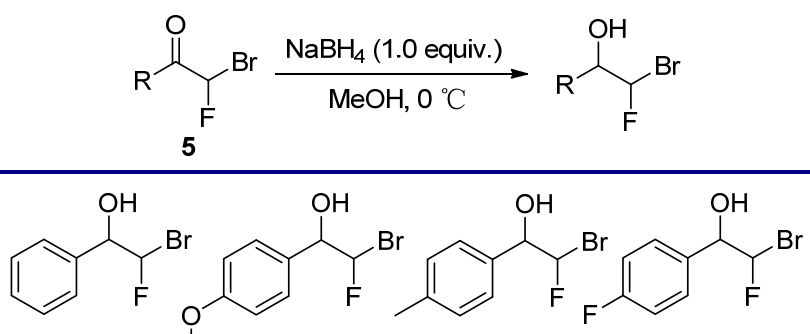

$5 a, 82 \%$

$5 \mathbf{b}, 83 \%$

$5 c, 81 \%$

5d, $85 \%$

${ }^{a}$ Reaction condition: $5(1 \mathrm{mmol}), \mathrm{NaBH}_{4}(1 \mathrm{mmol}), \mathrm{MeOH}(10 \mathrm{~mL})$ at $0{ }^{\circ} \mathrm{C}$ for $5 \mathrm{~min}$. Isolated yields.
最后，推测该反应的机理如 Scheme 4 所示. 硼氢化 钠中的氢负离子首先进攻羰基碳原子，接着从甲醇溶剂 中得到氢, 形成含二氟碘醇类化合物.

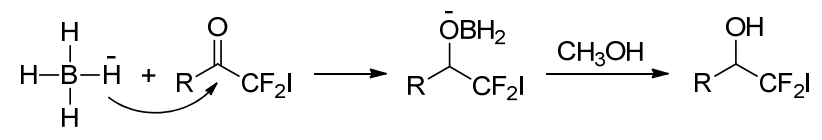

图式 4 可能的还原机理

Scheme 4 Proposed mechanism

\section{2 结论}

本文成功地探索出了一种简单有效地利用 $\mathrm{NaBH}_{4}$ 选择性还原 $\alpha, \alpha, \alpha$-碘二氟苯乙酮类化合物制备 $\beta, \beta, \beta$-二 氟碘-1-苯基乙醇类化合物的方法. 该方法反应温和，还 原选择性高, 产率高. 较其他的方法更为简单, 反应时 间大大缩短，效率更高，也从理论上对在偕二氟基团存 在情况下羰基与卤素基团的选择性还原情况作了补充. 该方法对 $\alpha, \alpha, \alpha$-碘二氟苯乙酮、 $\alpha, \alpha, \alpha$-溴二氟苯乙酮、 $\alpha, \alpha, \alpha$-氯二氟苯乙酮和 $\alpha, \alpha$-溴氟苯乙酮底物均有很高的 选择还原性，而对其 $\alpha$ 位卤原子无任何影响，使得用 $\beta$ 偕二氟取代醇类片段进一步与不同的底物反应，得到结 构复杂多样的新颖含氟化合物成为可能. 后期将以 
$\beta, \beta, \beta$-二氟碘-1-苯基乙醇类为砌块，与不同的底物反应 引入 $\beta$-偕二氟取代醇类片段, 得到结构复杂多样的新颖 含氟化合物, 并对目标产物进行生物活性篮选研究. 这 些工作正在进行中.

\section{3 实验部分}

\section{1 仪器与试剂}

核磁共振氢谱 $\left({ }^{1} \mathrm{H} N \mathrm{NMR}\right) 、$ 氟谱 $\left({ }^{19} \mathrm{~F} \mathrm{NMR}\right)$ 和碳谱 $\left({ }^{13} \mathrm{C}\right.$ NMR)是使用 Bruker 公司的 Bruker AVANCE3-500 型核 磁共振谱仪测得, 内标物是四甲基硅烷(TMS), 溶剂为 $\mathrm{CDCl}_{3}$; 高分辨质谱( $\mathrm{EI}$ )是通过 Finigann MAT8401 质谱 仪测定; 红外色谱(IR)是通过尼高力电公司的 Nicolet 380 测定. 实验所用试剂均为市售化学纯或分析纯试剂.

\section{2 实验方法}

\subsection{1 $\alpha, \alpha, \alpha$-磑二氟苯乙醇类化合物的制备}

在 $50 \mathrm{~mL}$ 的圆底烧瓶中, 将 $1 \mathrm{mmol}$ 的 $\alpha, \alpha, \alpha$-碘二氟 苯乙酮 1 或 $\alpha, \alpha$-溴氟苯乙酮 5 溶于 $10 \mathrm{~mL} \mathrm{MeOH}$ 中. 在 $0{ }^{\circ} \mathrm{C}$ 下, 缓慢加入 1.0 equiv. 的 $\mathrm{NaBH}_{4}$, 搅拌 $5 \mathrm{~min}$. 薄层 色谱(TLC)检测反应, 反应完全后加水淬灭, 旋除溶剂, 用二氯甲烷萃取 $(30 \mathrm{~mL} \times 3)$, 合并有机相, 无水硫酸钠 干燥, 旋干, 通过硅胶快速柱色谱法 [洗脱液: $V$ (石油 醚) $: V($ 乙酸乙酯 $)=20: 1$ ] 得到目标产物.

2,2-二氟-2-碘-1-苯基乙醇 $(2 \mathbf{a})^{[7]}$ : 淡黄色油状液体, 产率 94\%. ${ }^{1} \mathrm{H}$ NMR $\left(500 \mathrm{MHz}, \mathrm{CDCl}_{3}\right) \delta: 7.30 \sim 7.51(\mathrm{~m}$, $5 \mathrm{H}), 4.68(\mathrm{dd}, J=10.0,5.0 \mathrm{~Hz}, 1 \mathrm{H}), 2.56(\mathrm{~s}, 1 \mathrm{H}) ;{ }^{19} \mathrm{~F}$ NMR $\left(470 \mathrm{MHz}, \mathrm{CDCl}_{3}\right) \delta:-48.0(\mathrm{~d}, J=178.6 \mathrm{~Hz}, 1 \mathrm{~F})$, $-52.9(\mathrm{~d}, J=178.6 \mathrm{~Hz}, 1 \mathrm{~F}) ;{ }^{13} \mathrm{C}$ NMR $\left(125 \mathrm{MHz}, \mathrm{CDCl}_{3}\right)$ $\delta: 134.6,129.4,128.4,128.0,107.9\left(\mathrm{t},{ }^{1} J_{\mathrm{C}-\mathrm{F}}=315.0 \mathrm{~Hz}\right)$, $80.0\left(\mathrm{t},{ }^{2} J_{\mathrm{C}-\mathrm{F}}=25.0 \mathrm{~Hz}\right)$.

2,2-二氟-2-磑-1-(4-甲基苯基)乙醇(2b): 淡黄色油 状液体, 产率 93\%. ${ }^{1} \mathrm{H}$ NMR $\left(500 \mathrm{MHz}, \mathrm{CDCl}_{3}\right) \delta: 7.35$ (d, $J=5.0 \mathrm{~Hz}, 2 \mathrm{H}), 7.19$ (d, $J=10.0 \mathrm{~Hz}, 2 \mathrm{H}), 4.62$ (dd, $J=$ 15.0, $5.0 \mathrm{~Hz}, 1 \mathrm{H}), 2.72(\mathrm{~s}, 1 \mathrm{H}), 2.35$ (s, 3H); ${ }^{19} \mathrm{~F}$ NMR $\left(470 \mathrm{MHz}, \mathrm{CDCl}_{3}\right) \delta:-48.2(\mathrm{~d}, J=178.6 \mathrm{~Hz}, 1 \mathrm{~F})$, $-52.8(\mathrm{~d}, J=178.6 \mathrm{~Hz}, 1 \mathrm{~F}) ;{ }^{13} \mathrm{C} \mathrm{NMR}\left(125 \mathrm{MHz}, \mathrm{CDCl}_{3}\right)$ $\delta: 139.5,131.7\left(\mathrm{~d},{ }^{1} J_{\mathrm{C}-\mathrm{F}}=2.5 \mathrm{~Hz}\right), 129.2,127.9,108.1(\mathrm{t}$, $\left.{ }^{1} J_{\mathrm{C}-\mathrm{F}}=316.3 \mathrm{~Hz}\right), 79.9\left(\mathrm{t},{ }^{2} J_{\mathrm{C}-\mathrm{F}}=22.5 \mathrm{~Hz}\right), 21.4$; IR (KBr) $v: 3550,3474,3412,1637,1616,1401,1397,1667,1020$, 990, 935, 781, 736, $591 \mathrm{~cm}^{-1}$; HRMS (EI-TOF) calcd for $\mathrm{C}_{9} \mathrm{H}_{10} \mathrm{~F}_{2} \mathrm{IO}\left([\mathrm{M}+\mathrm{H}]^{+}\right)$297.9666, found 297.9665.

2,2-二氟-2-碘-1-(4-甲氧基苯基)乙醇 $(2 \mathbf{c})^{[7]}$ : 淡黄色 油状液体, 产率 $93 \% .{ }^{1} \mathrm{H}$ NMR $\left(500 \mathrm{MHz}, \mathrm{CDCl}_{3}\right) \delta: 7.39$ (d, $J=10.0 \mathrm{~Hz}, 2 \mathrm{H}), 6.91$ (d, $J=10.0 \mathrm{~Hz}, 2 \mathrm{H}), 4.63$ (dd, $J=10.0,5.0 \mathrm{~Hz}, 1 \mathrm{H}), 3.82(\mathrm{~s}, 3 \mathrm{H}), 2.65(\mathrm{~s}, 1 \mathrm{H}) ;{ }^{19} \mathrm{~F} \mathrm{NMR}$ $\left(470 \mathrm{MHz}, \mathrm{CDCl}_{3}\right) \delta:-48.4(\mathrm{~d}, J=178.6 \mathrm{~Hz}, 1 \mathrm{~F})$, $-53.0(\mathrm{~d}, J=178.6 \mathrm{~Hz}, 1 \mathrm{~F}) ;{ }^{13} \mathrm{C}$ NMR $\left(125 \mathrm{MHz}, \mathrm{CDCl}_{3}\right)$ $\delta: 160.2,129.4,128.7,113.9,108.5\left(\mathrm{t},{ }^{1} J_{\mathrm{C}-\mathrm{F}}=316.3 \mathrm{~Hz}\right)$, $79.6\left(\mathrm{t},{ }^{2} J_{\mathrm{C}-\mathrm{F}}=22.5 \mathrm{~Hz}\right), 55.5$.

2,2-二氟-2-碘-1-(4-氯苯基)乙醇 $(2 \mathbf{d})^{[7]}$ : 淡黄色油状 液体, 产率 $93 \% .{ }^{1} \mathrm{H}$ NMR $\left(500 \mathrm{MHz}, \mathrm{CDCl}_{3}\right) \delta: 7.40(\mathrm{dd}$, $J=20.0,10.0 \mathrm{~Hz}, 5 \mathrm{H}), 4.65(\mathrm{dd}, J=10.0,8.0 \mathrm{~Hz}, 1 \mathrm{H})$, $2.58(\mathrm{~s}, 1 \mathrm{H}) ;{ }^{19} \mathrm{~F}$ NMR $\left(470 \mathrm{MHz}, \mathrm{CDCl}_{3}\right) \delta:-48.4(\mathrm{~d}$, $J=183.3 \mathrm{~Hz}, 1 \mathrm{~F}),-53.4(\mathrm{~d}, J=183.3 \mathrm{~Hz}, 1 \mathrm{~F}) ;{ }^{13} \mathrm{C}$ NMR $\left(125 \mathrm{MHz}, \mathrm{CDCl}_{3}\right) \delta: 135.5,133.1,129.4,128.7,107.4(\mathrm{t}$, $\left.{ }^{1} J_{\mathrm{C}-\mathrm{F}}=316.3 \mathrm{~Hz}\right), 79.3\left(\mathrm{t},{ }^{2} J_{\mathrm{C}-\mathrm{F}}=23.8 \mathrm{~Hz}\right)$.

2,2-二氟-2-碘-1-(4-溴苯基)乙醇 $(2 \mathbf{e})^{[7]}$ : 淡黄色油状 液体, 产率 $93 \% .{ }^{1} \mathrm{H}$ NMR $\left(500 \mathrm{MHz}, \mathrm{CDCl}_{3}\right) \delta: 7.54(\mathrm{~d}$, $J=5.0 \mathrm{~Hz}, 2 \mathrm{H}), 7.36$ (d, $J=10.0 \mathrm{~Hz}, 2 \mathrm{H}), 4.63$ (dd, $J=$ 10.0, $5.0 \mathrm{~Hz}, 1 \mathrm{H}), 2.92(\mathrm{~s}, 1 \mathrm{H}) ;{ }^{19} \mathrm{~F}$ NMR $(470 \mathrm{MHz}$, $\left.\mathrm{CDCl}_{3}\right) \delta:-48.4(\mathrm{~d}, J=183.3 \mathrm{~Hz}, 1 \mathrm{~F}),-53.4(\mathrm{~d}, J=$ $178.6 \mathrm{~Hz}, 1 \mathrm{~F}) ;{ }^{13} \mathrm{C}$ NMR $\left(125 \mathrm{MHz}, \mathrm{CDCl}_{3}\right) \delta: 133.6$, $133.2,131.6,129.6,107.3\left(\mathrm{t},{ }^{1} J_{\mathrm{C}-\mathrm{F}}=317.5 \mathrm{~Hz}\right), 79.3(\mathrm{t}$, ${ }^{2} J_{\mathrm{C}-\mathrm{F}}=23.8 \mathrm{~Hz}$ ).

2,2-二氟-2-碘-1-(4-三氟甲基苯基)乙醇 $(2 \mathbf{f})^{[16]}$ : 淡黄 色油状液体, 产率 $86 \% .{ }^{1} \mathrm{H}$ NMR $\left(500 \mathrm{MHz}, \mathrm{CDCl}_{3}\right) \delta$ : $7.64(\mathrm{dd}, J=25.0,10.0 \mathrm{~Hz}, 4 \mathrm{H}), 7.32(\mathrm{t}, J=10.0 \mathrm{~Hz}, 1 \mathrm{H})$, $3.16(\mathrm{~s}, 1 \mathrm{H}) ;{ }^{19} \mathrm{~F}$ NMR $\left(470 \mathrm{MHz}, \mathrm{CDCl}_{3}\right) \delta:-48.3(\mathrm{~d}$, $J=183.3 \mathrm{~Hz}, 1 \mathrm{~F}),-53.5(\mathrm{~d}, J=183.3 \mathrm{~Hz}, 1 \mathrm{~F}),-62$. (s, $3 \mathrm{~F}) ;{ }^{13} \mathrm{C} \mathrm{NMR}\left(125 \mathrm{MHz}, \mathrm{CDCl}_{3}\right) \delta: 151.7(\mathrm{t}, J=27.5 \mathrm{~Hz})$, $130.2,128.9,128.4,128.3,127.1\left(\mathrm{q}, J_{\mathrm{C}-\mathrm{F}}=163.3,241.3\right.$ $\mathrm{Hz}), 114.2\left(\mathrm{t},{ }^{1} J_{\mathrm{C}-\mathrm{F}}=238.8 \mathrm{~Hz}\right)$.

2,2-二氟-2-硒-1-(4-氟苯基)乙醇 (2g)：淡黄色油状 液体, 产率 $82 \% .{ }^{1} \mathrm{H}$ NMR $\left(500 \mathrm{MHz}, \mathrm{CDCl}_{3}\right) \delta: 7.47(\mathrm{t}$, $J=5.0 \mathrm{~Hz}, 2 \mathrm{H}), 7.09$ (t, $J=10.0 \mathrm{~Hz}, 2 \mathrm{H}), 4.67$ (dd, $J=$ 10.0, $5.0 \mathrm{~Hz}, 1 \mathrm{H}), 2.94(\mathrm{~s}, 1 \mathrm{H}) ;{ }^{19} \mathrm{~F}$ NMR $(470 \mathrm{MHz}$, $\left.\mathrm{CDCl}_{3}\right) \delta:-48.5(\mathrm{~d}, J=183.3 \mathrm{~Hz}, 1 \mathrm{~F}),-53.4(\mathrm{~d}, J=$ $183.3 \mathrm{~Hz}, 1 \mathrm{~F}),-111.9$ (s, 1F); ${ }^{13} \mathrm{C}$ NMR $(125 \mathrm{MHz}$, $\left.\mathrm{CDCl}_{3}\right) \delta: 163.3\left(\mathrm{~d}, J_{\mathrm{C}-\mathrm{F}}=258.8 \mathrm{~Hz}\right), 129.8\left(\mathrm{~d},{ }^{3} J_{\mathrm{C}-\mathrm{F}}=8.8\right.$ $\mathrm{Hz}), 115.4\left(\mathrm{~d},{ }^{3} J_{\mathrm{C}^{-\mathrm{F}}}=21.3 \mathrm{~Hz}\right), 107.8\left(\mathrm{t},{ }^{2} J_{\mathrm{C}^{-\mathrm{F}}}=316.3 \mathrm{~Hz}\right)$, $79.3\left(\mathrm{t},{ }^{1} J_{\mathrm{C}-\mathrm{F}}=23.8 \mathrm{~Hz}\right)$; IR (KBr) v: $3447,1648,1630$, 1607, 1510, 1409, 1230, 1161, 1083, 992, 935, 836, 794, 698, $560 \mathrm{~cm}^{-1}$; HRMS (EI-TOF) calcd for $\mathrm{C}_{8} \mathrm{H}_{7} \mathrm{~F}_{3} \mathrm{IO}$ $\left([\mathrm{M}+\mathrm{H}]^{+}\right)$301.9416, found 301.9417 .

2,2-二氟-1-(4-氟苯基)乙醇 $(\mathbf{4 g})^{[17]}$ : 淡黄色油状液 体, 产率 9\%. ${ }^{1} \mathrm{H}$ NMR $\left(500 \mathrm{MHz}, \mathrm{CDCl}_{3}\right) \delta: 7.41$ (t, $J=$ $5.0 \mathrm{~Hz}, 2 \mathrm{H}), 7.09(\mathrm{t}, J=10.0 \mathrm{~Hz}, 2 \mathrm{H}), 5.73(\mathrm{td}, J=60.0$, $5.0 \mathrm{~Hz}, 1 \mathrm{H}), 4.82(\mathrm{td}, J=10.0,5.0 \mathrm{~Hz}, 1 \mathrm{H}), 2.53(\mathrm{~s}, 1 \mathrm{H})$. ${ }^{19} \mathrm{~F}$ NMR $\left(470 \mathrm{MHz}, \mathrm{CDCl}_{3}\right) \delta:-112.7(\mathrm{~s}, 1 \mathrm{~F}),-127.7$ $(\mathrm{AB}, J=305.5,286.7 \mathrm{~Hz}, 2 \mathrm{~F}) ;{ }^{13} \mathrm{C}$ NMR $(125 \mathrm{MHz}$, $\left.\mathrm{CDCl}_{3}\right) \delta: 163.1(\mathrm{~d}, J=246.3 \mathrm{~Hz}), 131.6,128.9(\mathrm{~d}, J=8.8$ 
$\mathrm{Hz}), 115.7,115.6\left(\mathrm{t},{ }^{1} J_{\mathrm{C}-\mathrm{F}}=243.8 \mathrm{~Hz}\right), 115.5,73.0(\mathrm{t}$, $\left.{ }^{2} J_{\mathrm{C}-\mathrm{F}}=25.0 \mathrm{~Hz}\right)$.

2,2-二氟-2-碘-1-(2-氟苯基)乙醇 (2h): 淡黄色油状 液体, 产率 $83 \% .{ }^{1} \mathrm{H}$ NMR $\left(500 \mathrm{MHz}, \mathrm{CDCl}_{3}\right) \delta: 7.64(\mathrm{t}$, $J=10.0 \mathrm{~Hz}, 1 \mathrm{H}), 7.40(\mathrm{td}, J=10.0,5.0 \mathrm{~Hz} 1 \mathrm{H}), 7.22(\mathrm{t}$, $J=10.0 \mathrm{~Hz}, 1 \mathrm{H}), 7.09$ (t, $J=10.0 \mathrm{~Hz}, 1 \mathrm{H}), 5.14(\mathrm{dd}, J=$ 10.0, $5.5 \mathrm{~Hz}, 1 \mathrm{H}), 3.03(\mathrm{~s}, 1 \mathrm{H}) ;{ }^{19} \mathrm{~F}$ NMR $(470 \mathrm{MHz}$, $\left.\mathrm{CDCl}_{3}\right) \delta:-49.3(\mathrm{dd}, J=178.6,5.0 \mathrm{~Hz}, 1 \mathrm{~F}),-53.3(\mathrm{dd}$, $J=178.6,5.0 \mathrm{~Hz}, 1 \mathrm{~F}),-116.6(\mathrm{dd}, J=15.0,5.0 \mathrm{~Hz}, 1 \mathrm{~F})$; ${ }^{13} \mathrm{C}$ NMR $\left(125 \mathrm{MHz}, \mathrm{CDCl}_{3}\right) \delta: 160.4(\mathrm{~d}, J=247.5 \mathrm{~Hz})$, 131.1 (d, $J=7.5 \mathrm{~Hz}), 129.2,124.3,115.5(\mathrm{~d}, J=21.3 \mathrm{~Hz})$, $106.5\left(\mathrm{t},{ }^{2} J_{\mathrm{C}-\mathrm{F}}=315.0 \mathrm{~Hz}\right), 74.0\left(\mathrm{t},{ }^{1} J_{\mathrm{C}-\mathrm{F}}=24.0 \mathrm{~Hz}\right) ; \mathrm{IR}$ (KBr) v: 3444, 1630, 1493, 1458, 1234, 1183, 1109, 992, 938, 757, 703, $526 \mathrm{~cm}^{-1}$; HRMS (EI-TOF) calcd for $\mathrm{C}_{8} \mathrm{H}_{7} \mathrm{~F}_{3} \mathrm{IO}\left([\mathrm{M}+\mathrm{H}]^{+}\right)$301.9416, found 301.9417 .

2,2-二氟-1-(2-氟苯基) 乙醇 (4h $)^{[18]}$ : 淡黄色油状液 体, 产率 8\%. ${ }^{1} \mathrm{H}$ NMR $\left(500 \mathrm{MHz}, \mathrm{CDCl}_{3}\right) \delta: 7.53(\mathrm{t}, J=$ $10.0 \mathrm{~Hz}, 1 \mathrm{H}), 7.37$ (dd, $J=10.0,5.0 \mathrm{~Hz}, 1 \mathrm{H}), 7.22$ (t, $J=$ $10.0 \mathrm{~Hz}, 1 \mathrm{H}), 7.10(\mathrm{t}, J=5.0 \mathrm{~Hz}, 1 \mathrm{H}), 5.91$ (td, $J=15.0$, $5.0 \mathrm{~Hz}, 1 \mathrm{H}), 5.21 \sim 5,16(\mathrm{~m}, 1 \mathrm{H}), 2.54(\mathrm{~s}, 1 \mathrm{H}) ;{ }^{19} \mathrm{~F} \mathrm{NMR}$ $\left(470 \mathrm{MHz}, \mathrm{CDCl}_{3}\right) \delta:-118.2(\mathrm{dd}, J=10.0,5.0 \mathrm{~Hz}, 1 \mathrm{~F})$, -127.5 (dd, $J=282.0,10.0 \mathrm{~Hz}, 1 \mathrm{~F}),-129.8(\mathrm{dd}, J=$ 282.0, $10.0 \mathrm{~Hz}, 1 \mathrm{~F}) ;{ }^{13} \mathrm{C} \mathrm{NMR}\left(125 \mathrm{MHz}, \mathrm{CDCl}_{3}\right) \delta: 160.3$ (d, $J=246.3 \mathrm{~Hz}), 130.6$ (d, $J=8.8 \mathrm{~Hz}), 129.9,129.0,128.6$ (d, $J=3.8 \mathrm{~Hz}), 124.5(\mathrm{~d}, J=3.8 \mathrm{~Hz}), 115.5(\mathrm{~d}, J=21.3$ $\mathrm{Hz}), 68.1\left(\mathrm{t},{ }^{2} J_{\mathrm{C}-\mathrm{F}}=23.8 \mathrm{~Hz}\right)$

2,2-二氟-2-磑-1-噻吩乙醇 (2i): 淡黄色油状液体, 产率 90\%. ${ }^{1} \mathrm{H}$ NMR $\left(500 \mathrm{MHz}, \mathrm{CDCl}_{3}\right) \delta: 7.39$ (dd, $J=$ 10.0, $5.0 \mathrm{~Hz}, 1 \mathrm{H}), 7.20$ (d, $J=5.0 \mathrm{~Hz}, 1 \mathrm{H}), 7.04$ (dd, $J=$ $10.0,5.0 \mathrm{~Hz}, 1 \mathrm{H}), 4.90(\mathrm{t}, J=10.0 \mathrm{~Hz}, 1 \mathrm{H}), 2.77(\mathrm{~s}, 1 \mathrm{H})$; ${ }^{19} \mathrm{~F}$ NMR $\left(470 \mathrm{MHz}, \mathrm{CDCl}_{3}\right) \delta:-49.5(\mathrm{~d}, J=183.3 \mathrm{~Hz}$, $1 \mathrm{~F}),-53.2(\mathrm{~d}, J=183.3 \mathrm{~Hz}, 1 \mathrm{~F}) ;{ }^{13} \mathrm{C}$ NMR $(125 \mathrm{MHz}$, $\left.\mathrm{CDCl}_{3}\right) \delta: 137.3,127.3(\mathrm{~d}, J=91.3 \mathrm{~Hz}), 106.8\left(\mathrm{t},{ }^{2} J_{\mathrm{C}-\mathrm{F}}=\right.$ $316.3 \mathrm{~Hz}), 77.0\left(\mathrm{t},{ }^{1} J_{\mathrm{C}-\mathrm{F}}=26.3 \mathrm{~Hz}\right)$; IR $(\mathrm{KBr}) v: 3386$, 2925, 2854, 1617, 1435, 1359, 1302, 1262, 1216, 1161, 1097, 1044, 983, 923, 857, 783, 707, $599 \mathrm{~cm}^{-1}$; HRMS (EI-TOF) calcd for $\mathrm{C}_{6} \mathrm{H}_{6} \mathrm{~F}_{2} \mathrm{IOS}\left([\mathrm{M}+\mathrm{H}]^{+}\right)$289.9074, found 289.9075.

2,2-二氟-2-磑-1-菜基乙醇 $(\mathbf{2} \mathbf{j})^{[16]}$ : 淡黄色油状液体, 产率 84\%. ${ }^{1} \mathrm{H}$ NMR $\left(500 \mathrm{MHz}, \mathrm{CDCl}_{3}\right) \delta: 8.00(\mathrm{~s}, 1 \mathrm{H})$, $7.95(\mathrm{~s}, 1 \mathrm{H}), 7.85(\mathrm{t}, J=10.0 \mathrm{~Hz}, 3 \mathrm{H}), 7.57 \sim 7.48(\mathrm{~m}, 3 \mathrm{H})$, $4.82(\mathrm{dd}, J=10.5,8.5 \mathrm{~Hz}, 1 \mathrm{H}), 2.72(\mathrm{~s}, 1 \mathrm{H}) ;{ }^{19} \mathrm{~F} \mathrm{NMR}$ $\left(470 \mathrm{MHz}, \mathrm{CDCl}_{3}\right) \delta:-47.8(\mathrm{~d}, J=178.6 \mathrm{~Hz}, 1 \mathrm{~F}),-$ $52.6(\mathrm{~d}, J=178.6 \mathrm{~Hz}, 1 \mathrm{~F}) ;{ }^{13} \mathrm{C} \mathrm{NMR}\left(125 \mathrm{MHz}, \mathrm{CDCl}_{3}\right) \delta$ : 132.6, $131.8,131.1\left(\mathrm{~d},{ }^{1} J_{\mathrm{C}-\mathrm{F}}=2.5 \mathrm{~Hz}\right), 127.3,127.1$, $126.8,126.7,125.8,125.4,123.8,106.9\left(\mathrm{t},{ }^{1} J_{\mathrm{C} \mathrm{F}}=316.9\right.$ $\mathrm{Hz}), 79.1\left(\mathrm{t},{ }^{2} J_{\mathrm{C}-\mathrm{F}}=23.1 \mathrm{~Hz}\right)$.

2,2-二氟-1-菜基乙醇 $\left(\mathbf{4 j} \mathbf{j}^{[17]}\right.$ : 淡黄色油状液体, 产 率 9\%. ${ }^{1} \mathrm{H}$ NMR $\left(500 \mathrm{MHz}, \mathrm{CDCl}_{3}\right) \delta: 7.95 \sim 7.83(\mathrm{~m}$, $4 \mathrm{H}), 7.53 \sim 7.46(\mathrm{~m}, 3 \mathrm{H}), 5.86(\mathrm{td}, J=55.0,5.0 \mathrm{~Hz}, 1 \mathrm{H})$, $5.01(\mathrm{td}, J=10.0,5.0 \mathrm{~Hz}, 1 \mathrm{H}), 2.05(\mathrm{~s}, 1 \mathrm{H}) ;{ }^{19} \mathrm{~F}$ NMR $(470$ $\left.\mathrm{MHz}, \mathrm{CDCl}_{3}\right) \delta:-127.3(\mathrm{AB}, J=338.4,286.7 \mathrm{~Hz}, 2 \mathrm{~F})$; ${ }^{13} \mathrm{C}$ NMR (125 MHz, $\left.\mathrm{CDCl}_{3}\right) \delta: 133.5,133.2,133.1,128.5$, $128.1,127.7,126.6,126.5,126.4,124.3,115.9\left(\mathrm{t},{ }^{1} J_{\mathrm{C}^{-\mathrm{F}}}=\right.$ $243.8 \mathrm{~Hz}), 73.8\left(\mathrm{t},{ }^{2} J_{\mathrm{C}-\mathrm{F}}=25.0 \mathrm{~Hz}\right)$.

1,1-二氟-1-碘-4-苯基丁-2-醇 $(\mathbf{2 k})^{[19]}$ : 淡黄色油状液 体, 产率 88\%. ${ }^{1} \mathrm{H}$ NMR $\left(500 \mathrm{MHz}, \mathrm{CDCl}_{3}\right) \delta: 7.38(\mathrm{t}, J=$ $15.0 \mathrm{~Hz}, 2 \mathrm{H}), 7.28(\mathrm{~d}, J=10.0 \mathrm{~Hz}, 3 \mathrm{H}), 3.40(\mathrm{~s}, 1 \mathrm{H})$, $3.03 \sim 2.97(\mathrm{~m}, 1 \mathrm{H}), 2.85 \sim 2.77(\mathrm{~m}, 2 \mathrm{H}), 2.20 \sim 2.10(\mathrm{~m}$, $1 \mathrm{H}), 2.03 \sim 1.88(\mathrm{~m}, 1 \mathrm{H}) ;{ }^{19} \mathrm{~F}$ NMR $\left(470 \mathrm{MHz}, \mathrm{CDCl}_{3}\right) \delta$ : -47.8 (dd, $J=225.6,9.4 \mathrm{~Hz}, 1 \mathrm{~F}),-52.2$ (dd, $J=225.6$, $9.4 \mathrm{~Hz}, 1 \mathrm{~F}) ;{ }^{13} \mathrm{C}$ NMR $\left(125 \mathrm{MHz}, \mathrm{CDCl}_{3}\right) \delta: 140.9,128.9$, 128.8, 126.7, $109.9\left(\mathrm{t},{ }^{1} J_{\mathrm{C}-\mathrm{F}}=316.3 \mathrm{~Hz}\right), 77.5\left(\mathrm{t},{ }^{2} J_{\mathrm{C}-\mathrm{F}}=\right.$ $23.8 \mathrm{~Hz}), 33.6,31.2$.

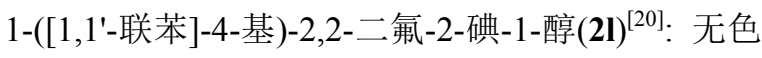
油状液体, 产率 $85 \% .{ }^{1} \mathrm{H}$ NMR $\left(500 \mathrm{MHz}, \mathrm{CDCl}_{3}\right) \delta$ : $7.58 \sim 7.55(\mathrm{~m}, 4 \mathrm{H}), 7.50(\mathrm{dd}, J=10.0,5.0 \mathrm{~Hz}, 2 \mathrm{H}), 7.41$ (t, $J=5.0 \mathrm{~Hz}, 2 \mathrm{H}), 7.33$ (t, $J=10.0 \mathrm{~Hz}, 1 \mathrm{H}), 4.82$ (dt, $J=$ 165.0, $10.0 \mathrm{~Hz}, 1 \mathrm{H}), 3.56(\mathrm{~s}, 1 \mathrm{H}) ;{ }^{19} \mathrm{~F}$ NMR $(376 \mathrm{MHz}$, $\left.\mathrm{CDCl}_{3}\right) \delta: \sim 47.9(\mathrm{dd}, J=181.0,7.7 \mathrm{~Hz}, 1 \mathrm{~F}),-55.67$ (dd, $J=162.4,6.8 \mathrm{~Hz}, 1 \mathrm{~F}) ;{ }^{13} \mathrm{C}$ NMR $\left(125 \mathrm{MHz}, \mathrm{CDCl}_{3}\right)$ $\delta: 142.3(\mathrm{~d}, J=12.5 \mathrm{~Hz}), 140.3(\mathrm{~d}, J=2.5 \mathrm{~Hz}), 133.7$, 133.4,129.0, 128.5 (d, $J=8.8 \mathrm{~Hz}), 127.7(\mathrm{~d}, J=1.3 \mathrm{~Hz})$, $127.2(\mathrm{~d}, J=2.5 \mathrm{~Hz}), 127.1(\mathrm{~d}, J=6.3 \mathrm{~Hz}), 79.8\left(\mathrm{t},{ }^{1} J_{\mathrm{C}^{-\mathrm{F}}}=\right.$ $23.8 \mathrm{~Hz}), 78.3\left(\mathrm{t},{ }^{2} J_{\mathrm{C}-\mathrm{F}}=25.0 \mathrm{~Hz}\right)$.

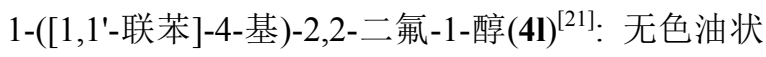
液体, 产率 $15 \% .{ }^{1} \mathrm{H}$ NMR $\left(500 \mathrm{MHz}, \mathrm{CDCl}_{3}\right) \delta: 7.62 \sim$ $7.57(\mathrm{~m}, 4 \mathrm{H}), 7.48 \sim 7.42(\mathrm{~m}, 4 \mathrm{H}), 7.37 \sim 7.34(\mathrm{~m}, 1 \mathrm{H})$, 5.79 (td, $J=60.0,5.0 \mathrm{~Hz}, 1 \mathrm{H}), 4.85(\mathrm{td}, J=10.0,5.0 \mathrm{~Hz}$, $1 \mathrm{H}), 2.67(\mathrm{~s}, 1 \mathrm{H}) ;{ }^{19} \mathrm{~F} \mathrm{NMR}\left(376 \mathrm{MHz}, \mathrm{CDCl}_{3}\right) \delta:-127.3$ (dd, $J=10.2,4.5 \mathrm{~Hz}, 1 \mathrm{~F}),-127.5$ (dd, $J=10.1,4.8 \mathrm{~Hz}$, 1F); ${ }^{13} \mathrm{C}$ NMR (125 MHz, $\mathrm{CDCl}_{3}$ ) $\delta: 142.0,140.5,134.8$, $128.9,127.6,127.5,127.4,127.2,115.9\left(\mathrm{t},{ }^{1} J_{\mathrm{C}-\mathrm{F}}=243.8\right.$ $\mathrm{Hz}), 73.5\left(\mathrm{t},{ }^{2} J_{\mathrm{C}-\mathrm{F}}=25.0 \mathrm{~Hz}\right)$.

2,2-二氟-2-碘-1-(4-苯氧基苯基)乙-1-醇(2m): 黄色 油状液体, 产率 $84 \% .{ }^{1} \mathrm{H}$ NMR $\left(500 \mathrm{MHz}, \mathrm{CDCl}_{3}\right) \delta: 7.40$ (d, $J=15.0 \mathrm{~Hz}, 2 \mathrm{H}), 7.33$ (t, $J=5.0 \mathrm{~Hz}, 2 \mathrm{H}), 7.12$ (t, $J=$ $5.0 \mathrm{~Hz}, 1 \mathrm{H}), 7.01(\mathrm{~d}, J=15.0 \mathrm{~Hz}, 2 \mathrm{H}), 6.98(\mathrm{~d}, J=5.0 \mathrm{~Hz}$, 2H), 4.59 (t, $J=10.0 \mathrm{~Hz}, 1 \mathrm{H}), 3.75(\mathrm{~s}, 1 \mathrm{H}), 3.70$ (t, $J=5.0$ $\mathrm{Hz}, 1 \mathrm{H}) ;{ }^{19} \mathrm{~F}$ NMR $\left(376 \mathrm{MHz}, \mathrm{CDCl}_{3}\right) \delta:-48.2(\mathrm{dd}, J=$ 
$180.5,7.8 \mathrm{~Hz}, 1 \mathrm{~F}),-52.9$ (dd, $J=180.5,10.4 \mathrm{~Hz}, 1 \mathrm{~F})$; ${ }^{13} \mathrm{C}$ NMR (125 MHz, $\left.\mathrm{CDCl}_{3}\right) \delta: 158.4,156.5,129.9,129.6$, $129.4,123.9,119.5,118.2,110.0\left(\mathrm{t},{ }^{1} J_{\mathrm{C}-\mathrm{F}}=254.9 \mathrm{~Hz}\right)$, $73.5\left(\mathrm{t},{ }^{2} J_{\mathrm{C}-\mathrm{F}}=22.5 \mathrm{~Hz}\right)$; IR (KBr) $v: 3451,1652,1631$, 1590, 1508, 1489, 1399, 1241, 1168, 1091, 990, 934, 873, $749,693,571 \mathrm{~cm}^{-1}$; HRMS (EI-TOF) calcd for $\mathrm{C}_{14} \mathrm{H}_{11} \mathrm{~F}_{2} \mathrm{I}-$ $\mathrm{O}_{2} \mathrm{Na}[\mathrm{M}+\mathrm{Na}]^{+}$398.9664, found 398.9665 .

2,2-二氟-1-(4-苯氧基苯基)乙-1-醇 $(\mathbf{4 m})^{[21]}$ : 无色油 状液体, 产率 $16 \% .{ }^{1} \mathrm{H}$ NMR $\left(500 \mathrm{MHz}, \mathrm{CDCl}_{3}\right) \delta: 7.38 \sim$ $7.33(\mathrm{~m}, 4 \mathrm{H}), 7.14 \sim 7.11(\mathrm{~m}, 1 \mathrm{H}), 7.03 \sim 7.00(\mathrm{~m}, 4 \mathrm{H})$, $5.75(\mathrm{td}, J=60.0,5.0 \mathrm{~Hz}, 1 \mathrm{H}), 4.80(\mathrm{td}, J=10.0,5.0 \mathrm{~Hz}$, 1H), 2.59 (s, $1 \mathrm{H}) ;{ }^{19} \mathrm{~F}$ NMR $\left(376 \mathrm{MHz}, \mathrm{CDCl}_{3}\right) \delta:-127.4$ $(\mathrm{d}, J=10.2 \mathrm{~Hz}, 1 \mathrm{~F}),-127.6(\mathrm{~d}, J=10.1 \mathrm{~Hz}, 1 \mathrm{~F}) ;{ }^{13} \mathrm{C}$ NMR $\left(125 \mathrm{MHz}, \mathrm{CDCl}_{3}\right) \delta: 158.1,156.7,130.4,129.9$, 128.7, 123.7, 119.30, 118.7, $115.8\left(\mathrm{t},{ }^{1} J_{\mathrm{C}-\mathrm{F}}=242.5 \mathrm{~Hz}\right)$, $73.2\left(\mathrm{t},{ }^{2} J_{\mathrm{C}-\mathrm{F}}=23.8 \mathrm{~Hz}\right)$.

(E)-1,1-二氟-1-碘-4-苯基丁-3-烯-2-醇(2n ${ }^{[22]}$ : 无色 油状液体, 产率 $60 \%$. ${ }^{1} \mathrm{H}$ NMR $\left(500 \mathrm{MHz}, \mathrm{CDCl}_{3}\right) \delta: 7.41$ (d, $J=10.0,2 \mathrm{H}), 7.35-7.28$ (m, 3H), 6.34 (dd, $J=15.0,5.0$ $\mathrm{Hz}, 1 \mathrm{H}), 6.16$ (ddd, $J=27.5,15.9,6.1 \mathrm{~Hz}, 1 \mathrm{H}), 4.17 \sim 4.11$ $(\mathrm{m}, 1 \mathrm{H}), 2.96(\mathrm{~s}, 1 \mathrm{H}) ;{ }^{19} \mathrm{~F}$ NMR $\left(376 \mathrm{MHz}, \mathrm{CDCl}_{3}\right) \delta$ : -48.8 (dd, $J=179.8,8.6 \mathrm{~Hz}, 1 \mathrm{~F}),-52.9$ (dd, $J=179.9$, $7.6 \mathrm{~Hz}, 1 \mathrm{~F}) ;{ }^{13} \mathrm{C} \mathrm{NMR}\left(125 \mathrm{MHz}, \mathrm{CDCl}_{3}\right) \delta: 136.1,135.6$, $128.8,128.7,127.0,122.6,108.2\left(\mathrm{t},{ }^{1} J_{\mathrm{C}-\mathrm{F}}=316.3 \mathrm{~Hz}\right)$, $78.6\left(\mathrm{t},{ }^{2} J_{\mathrm{C}-\mathrm{F}}=23.8 \mathrm{~Hz}\right)$.

(E)-1,1-二氟-4-苯基丁-3-烯-2-醇(4n) ${ }^{[23]}$ : 无色油状 液体, 产率 $24 \% .{ }^{1} \mathrm{H}$ NMR $\left(500 \mathrm{MHz}, \mathrm{CDCl}_{3}\right) \delta: 7.42 \sim$ $7.40(\mathrm{~m}, 1 \mathrm{H}), 7.35 \sim 7.32(\mathrm{~m}, 2 \mathrm{H}), 7.30 \sim 7.27(\mathrm{~m}, 1 \mathrm{H})$, $6.81(\mathrm{~d}, J=15.0 \mathrm{~Hz}, 1 \mathrm{H}), 6.21(\mathrm{dd}, J=15.0,5.0 \mathrm{~Hz}, 1 \mathrm{H})$, $5.72(\mathrm{td}, J=55.0,5.0 \mathrm{~Hz}, 1 \mathrm{H}), 4.49 \sim 4.44(\mathrm{~m}, 1 \mathrm{H}), 2.30$ (s, 1H); ${ }^{19} \mathrm{~F}$ NMR $\left(376 \mathrm{MHz}, \mathrm{CDCl}_{3}\right) \delta:-127.93$ (ddd, $J=285.9,56.3,10.7 \mathrm{~Hz}),-129.26$ (ddd, $J=286.0,55.8$, $10.3 \mathrm{~Hz}) ;{ }^{13} \mathrm{C}$ NMR $\left(125 \mathrm{MHz}, \mathrm{CDCl}_{3}\right) \delta: 135.8,134.9$, 128.7, 128.4, 126.8, 122.6, $115.5\left(\mathrm{t},{ }^{1} J_{\mathrm{C}-\mathrm{F}}=241.3 \mathrm{~Hz}\right)$, $72.2\left(\mathrm{t},{ }^{2} J_{\mathrm{C}-\mathrm{F}}=25.0 \mathrm{~Hz}\right)$.

2-澳-2,2-二氟-1-苯基乙-1-醇 $(20)^{[24]}$ : 淡黄色油状液 体, 产率 85\%. ${ }^{1} \mathrm{H}$ NMR $\left(500 \mathrm{MHz}, \mathrm{CDCl}_{3}\right) \delta: 7.49$ (t, $J=$ $10.0 \mathrm{~Hz}, 2 \mathrm{H}), 7.41 \sim 7.38(\mathrm{~m}, 3 \mathrm{H}), 5.15(\mathrm{dd}, J=20.0,10.0$ $\mathrm{Hz}, 1 \mathrm{H}), 2.89$ (s, $1 \mathrm{H}) ;{ }^{19} \mathrm{~F}$ NMR $\left(470 \mathrm{MHz}, \mathrm{CDCl}_{3}\right) \delta$ : -55.8 (dd, $J=202.1,9.4 \mathrm{~Hz}, 1 \mathrm{~F}),-59.2(\mathrm{dd}, J=202.1$, $9.4 \mathrm{~Hz}, 1 \mathrm{~F}) ;{ }^{13} \mathrm{C}$ NMR $\left(125 \mathrm{MHz}, \mathrm{CDCl}_{3}\right) \delta: 134.6,129.8$, $128.7,128.2,124.5\left(\mathrm{t},{ }^{1} J_{\mathrm{C}-\mathrm{F}}=307.5 \mathrm{~Hz}\right), 78.8\left(\mathrm{t},{ }^{2} J_{\mathrm{C}^{-\mathrm{F}}}=\right.$ $25.0 \mathrm{~Hz})$.

2-氯-2,2-二氟-1-苯基乙-1-醇 $(2 p)^{[25]}$ : 淡黄色油状液 体, 产率 93\%. ${ }^{1} \mathrm{H}$ NMR $\left(500 \mathrm{MHz}, \mathrm{CDCl}_{3}\right) \delta: 7.61(\mathrm{dd}$,
$J=10.0,5.0 \mathrm{~Hz}, 2 \mathrm{H}), 7.42 \sim 7.37$ (m, 3H), $5.22(\mathrm{~d}, J=5.0$ $\mathrm{Hz}, 1 \mathrm{H}), 3.38(\mathrm{~s}, 1 \mathrm{H}) ;{ }^{19} \mathrm{~F}$ NMR (376 MHz, $\left.\mathrm{CDCl}_{3}\right) \delta$ : 62.9 (dd, $J=165.0,7.4 \mathrm{~Hz}, 1 \mathrm{~F}),-64.7$ (dd, $J=165.0,8.5$ $\mathrm{Hz}, 1 \mathrm{~F}) ;{ }^{13} \mathrm{C}$ NMR $\left(125 \mathrm{MHz}, \mathrm{CDCl}_{3}\right) \delta: 135.1,129.8$, $129.5,128.1,103.4,84.8\left(\mathrm{~d},{ }^{1} J_{\mathrm{C}-\mathrm{F}}=1.3 \mathrm{~Hz}\right)$.

2-溴-2-氟-1-苯基乙-1-醇 $(\mathbf{5 a})^{[26]}$ : 淡黄色油状液体, 产率 82\%. ${ }^{1} \mathrm{H}$ NMR $\left(500 \mathrm{MHz}, \mathrm{CDCl}_{3}\right) \delta: 7.42 \sim 7.35(\mathrm{~m}$, $5 \mathrm{H}), 6.43$ (dd, $J=47.0,4.7 \mathrm{~Hz}, 1 \mathrm{H}), 4.99 \sim 4.95(\mathrm{~m}, 1 \mathrm{H})$, $2.74(\mathrm{~s}, 1 \mathrm{H}) ;{ }^{19} \mathrm{~F}$ NMR $\left(470 \mathrm{MHz}, \mathrm{CDCl}_{3}\right) \delta:-140.7(\mathrm{dd}$, $J=61.1,9.4 \mathrm{~Hz}, 1 \mathrm{~F}) ;{ }^{13} \mathrm{C} \mathrm{NMR}\left(125 \mathrm{MHz}, \mathrm{CDCl}_{3}\right) \delta$ : $137.0,129.3,128.9,127.2,98.6\left(\mathrm{~d},{ }^{1} J_{\mathrm{C}-\mathrm{F}}=256.3 \mathrm{~Hz}\right), 76.7$ $\left(\mathrm{d},{ }^{2} J_{\mathrm{C}-\mathrm{F}}=22.5 \mathrm{~Hz}\right)$.

2-溴-2-氟-1-(4-甲氧基苯基)乙-1-醇(5b): 无色油状 液体, 产率 $83 \% .{ }^{1} \mathrm{H}$ NMR $\left(500 \mathrm{MHz}, \mathrm{CDCl}_{3}\right) \delta: 7.32(\mathrm{dd}$, $J=10.0,5.0 \mathrm{~Hz}, 1 \mathrm{H}), 6.90(\mathrm{dd}, J=10.0,5.0 \mathrm{~Hz}, 1 \mathrm{H}), 6.40$ (ddd, $J=12.7,7.9,5.0 \mathrm{~Hz}, 5.0 \mathrm{~Hz}, 1 \mathrm{H}), 4.99 \sim 4.95(\mathrm{~m}$, 1H), 3.79 (s, 3H), $2.74(\mathrm{~s}, 1 \mathrm{H}) ;{ }^{19} \mathrm{~F}$ NMR (376 MHz, $\left.\mathrm{CDCl}_{3}\right) \delta:-140.9(\mathrm{dd}, J=49.0,9.2 \mathrm{~Hz}, 1 \mathrm{~F}) ;{ }^{13} \mathrm{C} \mathrm{NMR}$ $\left(125 \mathrm{MHz}, \mathrm{CDCl}_{3}\right) \delta: 160.1,128.6,128.3,114.1,98.4(\mathrm{~d}$, $\left.{ }^{1} J_{\mathrm{C}^{-\mathrm{F}}}=256.3 \mathrm{~Hz}\right), 76.2\left(\mathrm{~d},{ }^{2} J_{\mathrm{C}^{-\mathrm{F}}}=23.8 \mathrm{~Hz}\right), 55.35$; IR (KBr) $v: 3441,3386,1636,1613,1463,1305,1252,1177$, 1080, 1030, 833, 778, 737, 588, $571 \mathrm{~cm}^{-1}$; HRMS (EITOF) calcd for $\mathrm{C}_{9} \mathrm{H}_{11} \mathrm{BrFO}_{2}[\mathrm{M}+\mathrm{H}]^{+}$250.0884, found 250.0889 .

2-溴-2-氟-1-(对甲苯基)乙-1-醇(5c): 无色油状液体, 产率 81\%. ${ }^{1} \mathrm{H}$ NMR $\left(500 \mathrm{MHz}, \mathrm{CDCl}_{3}\right) \delta: 7.28(\mathrm{~d}, J=10.0$ $\mathrm{Hz}, 1 \mathrm{H}), 7.19$ (d, $J=5.0 \mathrm{~Hz}, 2 \mathrm{H}), 6.41$ (ddd, $J=47.5,12.5$, $5.0 \mathrm{~Hz}, 1 \mathrm{H}), 4.96 \sim 4.89(\mathrm{~m}, 1 \mathrm{H}), 2.86(\mathrm{~s}, 1 \mathrm{H}), 2.35(\mathrm{~s}$, $3 \mathrm{H}) ;{ }^{19} \mathrm{~F}$ NMR $\left(376 \mathrm{MHz}, \mathrm{CDCl}_{3}\right) \delta:-140.8(\mathrm{dd}, J=$ 49.7, $9.2 \mathrm{~Hz}, 1 \mathrm{~F}) ;{ }^{13} \mathrm{C}$ NMR (125 MHz, $\left.\mathrm{CDCl}_{3}\right) \delta: 138.9$, 133.8, 129.4, 126.9, $98.4\left(\mathrm{~d},{ }^{1} J_{\mathrm{C}-\mathrm{F}}=255.0 \mathrm{~Hz}\right), 76.4(\mathrm{~d}$, ${ }^{2} J_{\mathrm{C}-\mathrm{F}}=21.3 \mathrm{~Hz}$ ), 21.3; IR (KBr) v: 3404, 3030, 2962, 2923, 2857, 1615, 1515, 1415, 1261, 1181, 1082, 1031, 949, 802, 737, 682, $562 \mathrm{~cm}^{-1}$; HRMS (EI-TOF) calcd for $\mathrm{C}_{9} \mathrm{H}_{11} \mathrm{BrFO}[\mathrm{M}+\mathrm{H}]^{+}$234.0836, found 234.0839.

2-溴-2-氟-1-(4-氟苯基)乙-1-醇 $(\mathbf{5 d})$ : 淡黄色油状液 体, 产率 85\%. ${ }^{1} \mathrm{H}$ NMR $\left(500 \mathrm{MHz}, \mathrm{CDCl}_{3}\right) \delta: 7.39$ (t, $J=$ $10.0 \mathrm{~Hz}, 2 \mathrm{H}), 7.08$ (t, $J=10.0 \mathrm{~Hz}, 2 \mathrm{H}), 6.40$ (ddd, $J=47.5$, $17.5,5.0 \mathrm{~Hz}, 1 \mathrm{H}), 5.00 \sim 4.92(\mathrm{~m}, 1 \mathrm{H}), 2.86(\mathrm{~s}, 1 \mathrm{H}) ;{ }^{19} \mathrm{~F}$ NMR $\left(376 \mathrm{MHz}, \mathrm{CDCl}_{3}\right) \delta:-112.5$ (ddd, $J=14.6,9.1$, $5.6 \mathrm{~Hz}, 1 \mathrm{~F}),-141.4(\mathrm{dd}, J=49.2,9.6 \mathrm{~Hz}, 1 \mathrm{~F}) ;{ }^{13} \mathrm{C}$ NMR $\left(125 \mathrm{MHz}, \mathrm{CDCl}_{3}\right) \delta: 163.0(\mathrm{~d}, J=245.0 \mathrm{~Hz}), 129.2(\mathrm{~d}$, $J=8.8 \mathrm{~Hz}), 128.8(\mathrm{~d}, J=5.0 \mathrm{~Hz}), 115.6(\mathrm{~d}, J=125.0 \mathrm{~Hz})$, $98.1\left(\mathrm{~d},{ }^{1} J_{\mathrm{C}-\mathrm{F}}=256.3 \mathrm{~Hz}\right), 75.8\left(\mathrm{~d},{ }^{2} J_{\mathrm{C}-\mathrm{F}}=21.3 \mathrm{~Hz}\right), 21.3$; IR (KBr) v: 3443, 1647, 1631, 1608, 1511, 1405, 1401, 
1230, 691, $556 \mathrm{~cm}^{-1}$; HRMS (EI-TOF) calcd for $\mathrm{C}_{8} \mathrm{H}_{8} \mathrm{Br}-$ $\mathrm{F}_{2} \mathrm{O}[\mathrm{M}+\mathrm{H}]^{+}$237.0456, found 237.0459.

辅助材料(Supporting Information) 本文章中化合物的 核磁谱图和红外谱图可以免费从本刊网站(http://siocjournal.cn/)上下载.

\section{References}

[1] Nabi, N.; Agarwal, R.; Mukhopadhyay, H.; Banoo, H.; Nigam, N.; Nigam, N. J. Evol. Med. Dent. Sci. 2017, 6, 215.

[2] Zheng, L; Zhao, T.; Zou, X. Chin. J. New. Durgs 2015, 24, 361 (in Chinese).

(陈玲，赵天笑，邹栩，中国新药杂志, 2015, 24, 361.)

[3] Dayer, P.; Desmenles, J.; Collart, L. Drugs 1997, 53, 18.

[4] (a) Hiyama, T. Textbook of Organofluorine Compounds: Chemistry and Applications, Springer, New York, 2000.

(b) Gouverneur, V.; Muller, K. Textbook of Fluorine in Pharmaceutical and Medicinal Chemistry: From Biophysical Aspects to Clinical Applications, Imperial College Press, London, 2012.

(c) Huchet, B.; Kuhn, B.; Wagner, N.; Zimmerli, E. M.; Carreira, K. J. Med. Chem. 2015, 58, 9041 .

(d) Gillis, E. P.; Eastman, K. J.; Hill, M. D.; Donnelly, D. J.; Meanwell, N. A. J. Med. Chem. 2015, 58, 8315.

[5] (a) Wang, J.; Sánchez-Roselló, M.; Aceña, J. L.; del Pozo, C.; Sorochinsky, A. E.; Fustero, S.; Soloshonok, V. A.; Liu, H. Chem. Rev. 2014, 114, 2432.

(b) Müller, K.; Faeh, C.; Diederich, F. Science 2007, 317, 1881.

(c) Fu, X. P.; Xiao, Y. L.; Zhang, X. Chin. J. Chem. 2018, 36, 143.

(d) Qi, Q. X.; Zi, Y. Z.; Ling, C. L.; Chuan, F. N.; Jin, B. H. Angew. Chem. Int. Ed. 2019, 58, 6405 .

[6] Jagodzinska, M.; Huguenot, F.; Zanda, M. Tetrahedron 2007, 63, 2042.

[7] Yan, C. Z.; Bing, G.; Jin, B. H. J. Am. Chem. Soc. 2012, 134, 5790.

[8] Kosobokov, M. D.; Levin, W.; Struchkova, M. I.; Dilman, A. D. Org. Lett. 2014, 16, 3784.

[9] Aikawa, K.; Yoshida, S.; Kondo, D.; Asai, Y.; Mikami, K. Org. Lett.
2015, 17, 5108.

[10] (a) Wang, J. X.; Wu, J. J.; Chen, H.; Zhang, S. W.; Wu, F. H. Chin. Chem Lett. 2015, 26, 1381.

(b) Wang, D. F.; Wu, J. J.; Huang, J. W.; Liang, J. Q.; Peng, P.; Chen, H.; Wu, F. H. Tetrahedron 2017, 73, 3478

(c) Chen, H.; Wang, J. X.; Wu, J. J.; Kuang, Y. J.; Wu, F. H. J. Fluorine Chem. 2017, 200, 41.

(d) Peng, P.; Wu, J. J.; Wu, F. H. RSC Adv. 2017, 7, 56034.

(e) Liang, J. Q.; Wu, J. J.; Wu, F. H. Adv. Synth. Catal. 2018 , 360(11), 2221.

[11] Miyachi, N.; Yanagawa, Y.; Iwasaki, H. Tetrahedron Lett. 1993, 34, 8267.

[12] Yang, F. C.; Yang, W. C.; Yang, C.; Wang, H. R.; Sun, K.; Luo, Y. J. Label Compd. Radiopharm. 2016, 59, 313.

[13] Toda, F.; Tanaka, K.; Tange, H. J. Chem. Soc., Perkin Trans. 1 1989, 1555 .

[14] Bajwa, N.; Jennings, M. P. J. Org. Chem. 2008, 73, 3638.

[15] Renata, H.; Zhou, Q. H.; Dünstl, G.; Felding, J.; Merchant, R. R.; Baran, P. S. J. Am. Chem. Soc. 2015, 137, 1330.

[16] Miao, W. J.; Ni, C. F.; Zhao, Y. C.; Jin, B. H. Org Lett. 2016, 18, 2766.

[17] Deng, Z. Y.; Lin, J. H.; Ji, C.; Xiao, J. C. Org Lett. 2016, 18. 3206

[18] Takamasa, F.; Toshinori, H. JP 05230075, 1993.

[19] Panferova, L. I.; Struchkova, M. I.; Dilman, A. D. Synthesis 2017, $49(18), 4124$.

[20] Wen, X. L.; Qing, J. L.; Ji, L. L.; Hong, G. W. Angew. Chem. Int. Ed. 2018, 57(50), 16544.

[21] Dilman, A.; Panferova, L.; Struchkova, M. Synthesis 2017, 49(18), 4124.

[22] Panferova, L. I.; Struchkova, M. I.; Dilman, A. D. Eur. J. Org. Chem. 2018, 27, 3834.

[23] Zhao, Y.; Huang, W.; Zheng, J.; Jin, B. H. Org Lett. 2011, 13(19), 5342.

[24] Capriel, P.; Binsch, G. Tetrahedron 1979, 35(22), 2661.

[25] Peng, P.; Wu, J.; Liang, J.; Zhang, T.; Huang, J.; Wu, F. RSC Advances. 2017, 7(88), 56034.

[26] Bergmann, F.; Kalmus, A.; Breuer, E. J. Am. Chem. Soc. 1958, 80, 4540.

(Li, L.; Fan, Y.) 